\title{
Uso de la embolización de la arteria meníngea media en recidiva de hematoma subdural crónico
}

\section{Use of embolization of the middle meningeal artery in chronic subdural hematoma recurrence}

Correspondencia Giancarlo Saal-Zapata gian_carlo1987@hotmail.com

\section{Recibido: 09/09/2020}

Arbitrado por pares

Aprobado: $12 / 11 / 2020$

Citar como: Vargas-Urbina JF, Saal-Zapata G, Rodriguez-Varela $R$. Uso de la embolización de la arteria meníngea media en recidiva de hematoma subdural crónico. Acta Med Peru. 2020;37(4):511-7. doi: https://doi.org/10.35663/ amp.2020.374.1825

\author{
John F. Vargas-Urbina ${ }^{1, a}$, Giancarlo Saal-Zapata²,b, Rodolfo Rodriguez-Varela2,b \\ Departamento de Neurocirugía, Hospital Nacional Guillermo Almenara Irigoyen. Lima, Perú. \\ Departamento de Neurocirugía, Servicio de Neurocirugía Endovascular, Hospital Nacional Guillermo Almenara Irigoyen. \\ Lima, Perú. \\ Médico cirujano; ${ }^{\mathrm{b}}$ médico cirujano especialista en Neurocirugía
}

\section{RESUMEN}

El hematoma subdural crónico (HSDC) es una patología neuroquirúrgica frecuente, con una recurrencia de hasta el $30 \%$ de los casos. La embolización de la arteria meníngea media (AMM) es una estrategia de manejo adyuvante para disminuir la recidiva, especialmente en pacientes con factores de riesgo para recurrencia, y tiene una baja tasa de complicaciones. Presentamos el caso de un varón de 83 años con antecedente de anticoagulación, que es admitido en centro sanitario por presentar hemiparesia derecha, disartria y alteración de la marcha, con un tiempo de enfermedad de una semana. Se realizó trepanación y evacuación del HSDC sin complicaciones. Un mes después retornó por recidiva del HSDC, por lo que se realizó trepanación y evacuación adecuada del HSDC, seguida de embolización de la AMM ipsilateral. El control a los dos meses mostró ausencia de recidiva, con mejoría neurológica del paciente. Concluimos que la embolización de la AMM fue un tratamiento adyuvante eficaz para disminuir la recidiva del HSDC y podría estar indicada en pacientes con factores de riesgo asociados a la recurrencia.

Palabras clave: Hematoma subdural crónico; Arterias meníngeas; Embolización; Recidiva; Trepanación (fuente: DeCS BIREME).

\begin{abstract}
Chronic subdural hematoma (CSDH) is a frequent neurosurgical condition, which may develop recurrence in up to $30 \%$ of all cases. Embolization of the middle meningeal artery (MMA) is an adjuvant management strategy for reducing recurrence, especially in patients with risk factors, and it has a low complication rate. We present the case of an 83-year old male with history of anticoagulation, who was admitted in a healthcare facility because of right hemiparesis, dysarthria and gait impairment, and he had been sick for one week. A trepanation and CSDH evacuation were uneventfully performed. One month later, the patient was brought back because of CSDH recurrence, so another trepanation and adequate CSDH evacuation were performed, followed by embolization of the ipsilateral MMA. A control assessment after two months revealed absence of recurrence and neurological improvement. We conclude that MMA embolization was an efficacious adjuvant therapy aiming to reduce CSDH recurrence, and this procedure may be indicated in patients with risk factors for recurrence.
\end{abstract}

Keywords: Hematoma, subdural, chronic; Meningeal arteries; Embolization; Recurrence; Trephining (source: MeSH NLM). 


\section{INTRODUCCIÓN}

El hematoma subdural crónico (HSDC) es una colección de productos de degradación sanguíneos localizada en el espacio subdural; es decir, entre la superficie cerebral y la duramadre ${ }^{[1-4]}$. Es una de las patologías más frecuentes en la consulta neuroquirúrgica, con una frecuencia de recurrencia de hasta el $30 \%$, vinculada a la fragilidad de la población mayor de edad ${ }^{[1,2,4,5]}$.

La embolización de la arteria meníngea media (AMM) es una estrategia de manejo para prevenir la recurrencia del HSDC luego de la evacuación ${ }^{[6]}$. Este procedimiento consiste en cateterizar la arteria carótida externa, luego, con un microcatéter, navegar por la AMM e inyectar sustancias embolizantes líquidas 0 también partículas. La fisiopatología del tratamiento se basa en la neoproliferación vascular de la membrana externa de la duramadre debido a cambios inflamatorios, los cuales pueden generar nuevos hematomas por microsangrados de los neovasos inmaduros a partir de la irrigación proveniente de la AMM. Además, existe un mecanismo adicional debido a la retención de contraste en la colección subdural luego de la embolización ${ }^{[5,7]}$.

Este procedimiento es una terapia adyuvante útil en el manejo del HSDC que tiene efecto de masa o desviación de línea media ${ }^{[1,5]}$ y es una alternativa atractiva como procedimiento único en pacientes que son pobres candidatos a una cirugía más invasiva ${ }^{[5]}$, que presentan poca sintomatología neurológica, que tienen una pobre situación de salud previa o cuando se trata de una recidiva del HSDC ${ }^{[2]}$, presentando buenos resultados en casos sin evacuación del HSDC ${ }^{[7]}$.

En la actualidad, la embolización de la AMM es una técnica novedosa, aunque requiere de tecnología para su realización, por lo que en nuestro medio su uso no se ha generalizado. Presentamos el primer caso exitoso de una embolización de la AMM por hematoma subdural crónico recidivado.

\section{REPORTE DE CASO}

Varón de 83 años con antecedente médico de trombosis venosa profunda en miembro inferior derecho, en tratamiento con warfarina, con antecedente quirúrgico de resección transuretral por hiperplasia benigna de próstata y cirugía láser por várices, sin alergia a medicamentos y sin antecedente de trauma. Ingresó al hospital con un tiempo de enfermedad de una semana, con hemiparesia derecha progresiva asociado a disartria y alteración de la marcha.

En nuestra institución se le realizó una tomografía espiral multicorte (TEM) cerebral sin contraste donde se evidenció una imagen hipodensa hemisférica izquierda en forma de semiluna de $20 \mathrm{~mm}$ de grosor, con desviación de línea media de $10 \mathrm{~mm}$; además, presentaba otra colección de iguales características a nivel del parietal derecho, siendo catalogado como un hematoma subdural crónico (HSDC) Nakaguchi homogéneo Markwalder II. Se programó por emergencia luego de corregir su INR y se le realizó una trepanación parietal izquierda, con evacuación adecuada del HSDC. La TEM posoperatoria mostró recuperación de la línea media y un pequeño sangrado por descompresión a nivel subdural derecho. (Figura 1).

Un mes después de la cirugía presentó cefalea global opresiva asociada a hemiparesia derecha, disartria y mareos. Se le realizó una TEM cerebral sin contraste donde se mostró una colección hemisférica izquierda en la misma localización que el HSDC, de $24 \mathrm{~mm}$ de grosor, con desviación de línea media de $8 \mathrm{~mm}$ y escaso edema hemisférico izquierdo; fue catalogado como HSDC hemisférico izquierdo recidivado Nakaguchi homogéneo Markwalder II, con un componente subagudo. No se identificó ninguna colección yuxtadural en el hemisferio derecho. El paciente había reiniciado su consumo de warfarina una semana antes y su INR era de 1,8, el cual fue corregido. Los exámenes de laboratorio mostraron un hemograma dentro de límites normales (hemoglobina: $13 \mathrm{~g} / \mathrm{dl}$, leucocitos: 7500 células $/ \mathrm{mm}^{3}$, plaquetas: 250000 ), una creatinina de $0,8 \mathrm{mg} / \mathrm{dl}$ y un examen de orina normal.

Se decidió programar por emergencia una trepanación parietal izquierda utilizando el agujero previo, lográndose una adecuada evacuación del hematoma, sin complicaciones agudas y restablecimiento de la línea media (Figura 2).

Después de la trepanación, se decidió embolizar la AMM izquierda para disminuir la probabilidad de recidiva (Figura 3). Este procedimiento se realizó bajo anestesia general, se colocó un introductor $6 \mathrm{~F}$ en la arteria femoral derecha y se avanzó un catéter guía $6 \mathrm{~F}$ hasta arteria carótida externa izquierda. Luego se navegó con microcatéter punta desprendible Sonic 1,5F apoyados con microguía Hybrid 007 hacia la arteria maxilar interna izquierda y hacia la AMM. Se logró identificar la entrada de la arteria a través del agujero espinoso y las ramas frontoparietal y temporal/escamosa. Se decidió embolizar totalmente la rama frontoparietal con sustancia embolizante Squid 18. La angiografía de control mostró embolización total de la rama.

En el periodo posoperatorio, el paciente recuperó el déficit motor y desaparecieron la disartria y mareos. En el segundo día posoperatorio se retiraron drenes subdurales sin complicaciones y se le dio de alta al día siguiente. Se le realizó control clínico y tomográfico a los dos meses, en donde se encontró que el paciente presentaba una escala de Rankin modificado de 0 y sin déficit motor. En la TEM de control no se evidenciaron colecciones yuxtadurales ni otras lesiones asociadas (Figura 4).

\section{DISCUSIÓN}

Este es el primer caso de una embolización de la AMM como tratamiento coadyuvante en un paciente con HSDC recidivado, lo cual agrega una alternativa terapéutica en este grupo de pacientes. A pesar de que el tratamiento fue realizado luego de dos cirugías de trepanación, éste ayudó a disminuir la recurrencia de esta patología. 


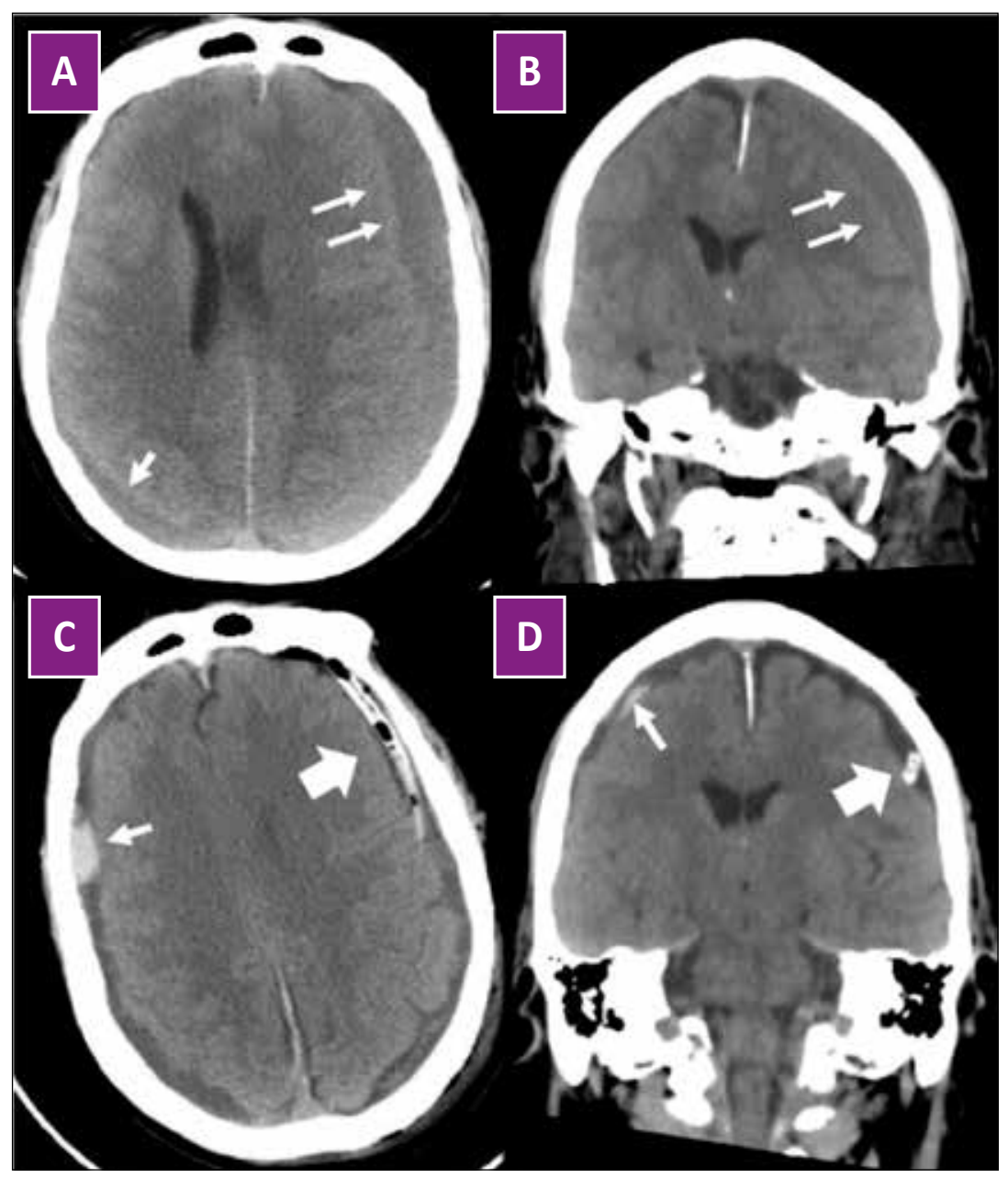

Figura 1. Tomografía espiral multicorte cerebral sin contraste. A y B: Corte axial y coronal, donde se evidencia una imagen hipodensa en forma de semiluna en región hemisférica izquierda (flechas dobles) compatible con un hemorragia subdural -catalogada como crónica (HSDC) por el relato clínico-, con desviación de línea media, y otra colección similar en región parietal derecha (flecha pequeña). C y D: Corte axial y coronal posoperatorio donde se evidencia adecuada evacuación del HSDC izquierdo, con escaso neumoencéfalo posquirúrgico y dren subdural en posición adecuada (flecha grande), con un hematoma subdural agudo frontal pequeño por descompresión (flecha pequeña).

Los HSDC tienen una incidencia por encima de 58,1 por cada 100000 pacientes/año en el grupo etario de mayores de 65 años y generalmente requieren manejo neuroquirúrgico, siendo tratados con frecuencia mediante un agujero de trépano y con un rango de recurrencia entre $11-37 \%{ }^{[3,4,8]}$. Los resultados del tratamiento son generalmente buenos, aunque puede haber recurrencia hasta en el $30 \%$ de los $\operatorname{casos}^{[1,3,4,9]}$.

La irrigación y el drenaje de un HSDC recurrente es todavía un tratamiento eficiente; no obstante, algunos pacientes tienen múltiples recurrencias, las cuales está asociadas a coagulopatías, atrofia cerebral y uso de fármacos anticoagulantes ${ }^{[1,8,10,11]}$. Un metaanálisis encontró que los pacientes con tratamiento anticoagulante tenían dos veces más riesgo de recurrencia comparado con los pacientes que no toman estos medicamentos, mientras que otro estudio indicaba que la terapia antiplaquetaria aumentaba cuatro veces el riesgo de recurrencia ${ }^{[8-12]}$.
Nuestro paciente tenía 83 años, lo que lo colocaba en un grupo de riesgo, con potenciales complicaciones posoperatorias; además, tenía el antecedente de consumir warfarina, características que aumentaban la posibilidad de recidiva. Inicialmente se le realizó una trepanación y evacuación del hematoma, que es el tratamiento estándar; luego, por presentar recidiva del hematoma, realizamos una nueva evacuación quirúrgica seguida de la embolización de la AMM con el fin de disminuir el riesgo de recurrencia.

A pesar de que la fisiopatología aún no es clara, se considera que el HSDC es causado por el daño de las venas puente, ya sea directo (debido a un trauma craneal menor) o indirecto (por la ruptura de una membrana aracnoidea de un higroma en expansión). Su formación se debe a una serie de mecanismos complejos que se iniciarían por la separación de la capa de células fronterizas en la duramadre, lo cual generaría respuestas curativas que incluyen la 


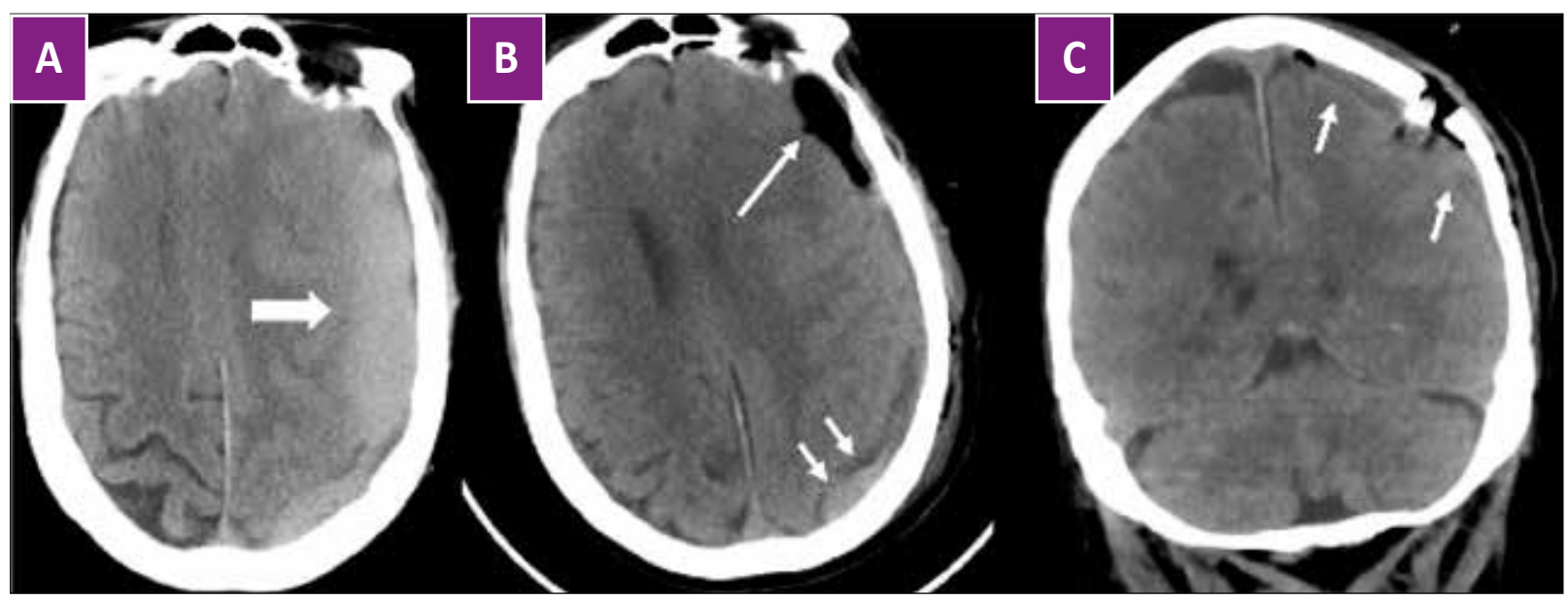

Figura 2. Tomografía espiral multicorte (TEM) cerebral sin contraste de control, luego de primera y segunda cirugía. A: TEM cerebral sin contraste de control luego de primera cirugía en la que se evidencia colección subdural en región hemisférica izquierda, que fue compatible con hematoma subdural crónico (HSDC) recidivado (flecha grande); el cual fue reoperado. B y C: Control tomográfico luego de segunda cirugía en el que se identifica neumoencéfalo escaso (flecha grande) y escaso HSDC residual inmediato (flechas pequeñas).

proliferación celular en el borde dural, la formación de tejido de granulación y depósito de macrófagos ${ }^{[1]}$. La migración de células inflamatorias y fibroblastos hacia la duramadre formaría una membrana que encapsula la colección subdural ${ }^{[7]}$. Finalmente, la inflamación local llevaría a hiperfibrinólisis del coágulo y producción de factores angiogénicos que pueden inducir neovascularización, que al romperse generan el hematoma ${ }^{[3,4,6-8]}$. Los hallazgos histológicos muestran pequeños vasos penetrantes desde la arteria meníngea media a través de la duramadre y conecta con neovasos en la membrana parietal. La ruptura de los neovasos puede generar el crecimiento del hematoma. La AMM juega un rol importante en la formación de un hematoma recurrente desde los neovasos ${ }^{[12]}$.

En el año 2000, Mandai presentó por primera vez a la embolización de la AMM para tratar de manera satisfactoria un HSDC recurrente ${ }^{[13]}$. Esta técnica contribuye a la teoría de que el crecimiento y la persistencia del HSDC es por un proceso patológico arterial, a diferencia de la naturaleza venosa que se ve en un hematoma subdural agudo ${ }^{[7]}$. Haldrup encontró que la tasa de recurrencia en los HSDC primarios embolizados fue del $4,1 \%$, mientras que para los recurrentes fue del $2,4 \%$, comparado con la tasa de recurrencia del $11,7 \%$ en los pacientes con trepanación, $19,4 \%$ en los pacientes con craneotomía y $28,1 \%$ en los pacientes con twist drill ${ }^{[1]}$.

Un estudio reportó un grupo de cinco pacientes con manejo inicial de embolización de la AMM en lugar de la cirugía, en pacientes en los que falló el manejo médico, y en el que todos experimentaron una reducción en el tamaño del hematoma, resolución de los síntomas, sin recurrencias ni complicaciones, sin necesidad de terminar en cirugía ${ }^{[14]}$.

En una revisión sistemática realizada por Court se reportó que la embolización de la AMM se reservaba sólo a los pacientes sintomáticos leves y que la mayoría era considerada como un tratamiento adyuvante. El $33 \%$ de las embolizaciones fueron realizadas antes del drenaje quirúrgico y $33 \%$ luego del mismo procedimiento, en ambos casos, en pacientes que tuvieron de una a tres recurrencias; además, hubo recurrencia en el 5,3\% de los casos luego de la embolización ${ }^{[12]}$. En otro estudio se encontró que el grupo de pacientes sometidos a embolización tenían menores frecuencias de recurrencia y reexpansión cerebral más temprana ${ }^{[7]}$.

Los hallazgos angiográficos son característicos en pacientes con HSDC. Link et al. identificaron estructuras con la apariencia de vasos con patrón de "lana de algodón", presentes en las ramas distales de la AMM, las mismas que, según Hashimoto et al. serían a la neovasculatura inmadura de la membrana subdural ${ }^{[15]}$. Por otra parte, se ha descrito que luego del procedimiento la colección subdural se nota más hiperdensa, lo cual se explicaría por el paso del contraste a través de los vasos arteriales que nacen de la AMM producto de la neovascularización, condición que haría a la zona vulnerable a hemorragias y con posibilidad de recurrencias ${ }^{[5,7]}$.

Los pacientes que padecen de esta patología son frecuentemente adultos mayores, por lo que la embolización puede tener complicaciones embólicas distales. Por eso, realizar una angiografía de la AMM antes de la embolización es importante para evaluar potenciales anastomosis "peligrosas" entre la arteria carótida externa (ACE) y la arteria carótida interna $(\mathrm{ACl})^{[1,16]}$. Court et al. en su revisión sistemática evidenciaron que la embolización de la AMM no presentó complicaciones asociadas en todos los pacientes analizados, sólo en dos artículos se encontraron complicaciones indirectas, que representaron el $3,6 \%$ del total de pacientes evaluados ${ }^{[12]}$.

Este reporte tiene algunas limitaciones. Incluimos uno solo caso, lo que hace imposible sacar conclusiones estadísticas robustas; 
además, en el medio hay una falta de reproducción de este procedimiento en otras instituciones debido a la ausencia de un angiógrafo y materiales embolizantes adecuados.

Se requieren mayores estudios prospectivos, en pacientes seleccionados para establecer a la embolización de la AMM como un tratamiento en el manejo del HSDC, ya sea como primera línea o como tratamiento coadyuvante en caso de recidiva.

Se concluye que la embolización de la AMM fue en nuestro caso un tratamiento adyuvante eficaz para disminuir la recurrencia del hematoma subdural crónico. Este procedimiento sería recomendado en pacientes con factores de riesgo asociados a recurrencia. Existiría también evidencia de un buen resultado de la embolización de la AMM como tratamiento único en pacientes seleccionados, aunque se requieren aún más estudios prospectivos que lo recomienden.

Contribuciones de autoría: JV-U y GS-Z concibieron y diseñaron la investigación, recolectaron los datos y redactaron y aprobaron la versión final del artículo. JV-U analizó los datos y redacto las tablas y gráficos; RR-V revisó críticamente el artículo. Los autores se responsabilizan por el contenido del artículo y se comprometen a responder adecuadamente las preguntas que pudieran ser necesarias para garantizar la precisión de los datos e integridad de cualquier parte de su investigación.
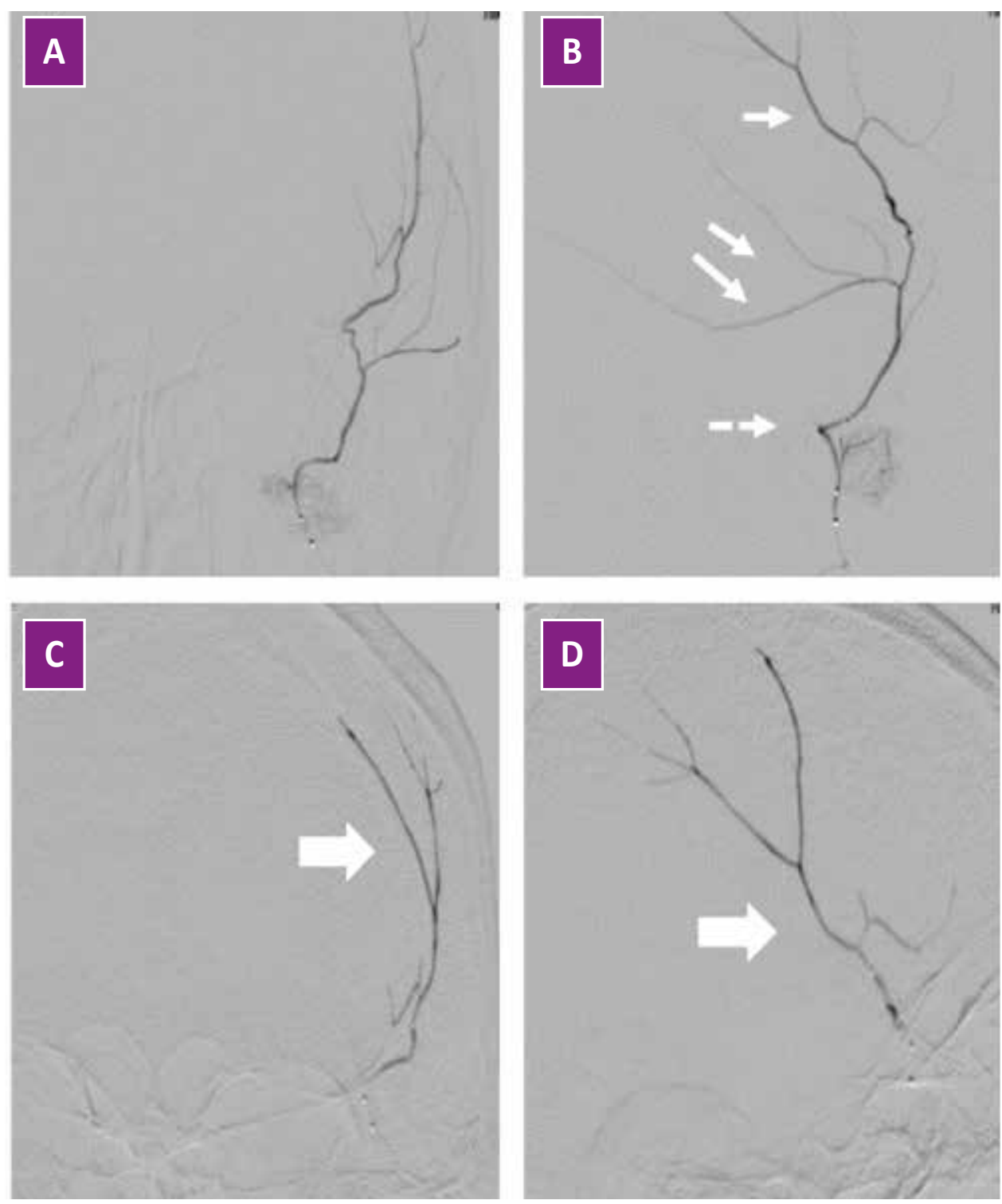

Figura 3. A y B: Angiografía ultraselectiva de arteria meningea media (AMM) izquierda en incidencia anteroposterior y lateral donde se visualizan las ramas frontoparietal (flecha única) y temporal/escamosa (flechas dobles). Se observa el ingreso de la AMM a través del agujero espinoso (flecha punteada). No se visualiza la rama escamosa. Cy D: Embolización con Squid $18{ }^{\circledR}$ de la rama frontoparietal de la AMM izquierda (flecha grande) con reflujo controlado por encima de la rama temporal en vista frontal (C) y lateral (D). 


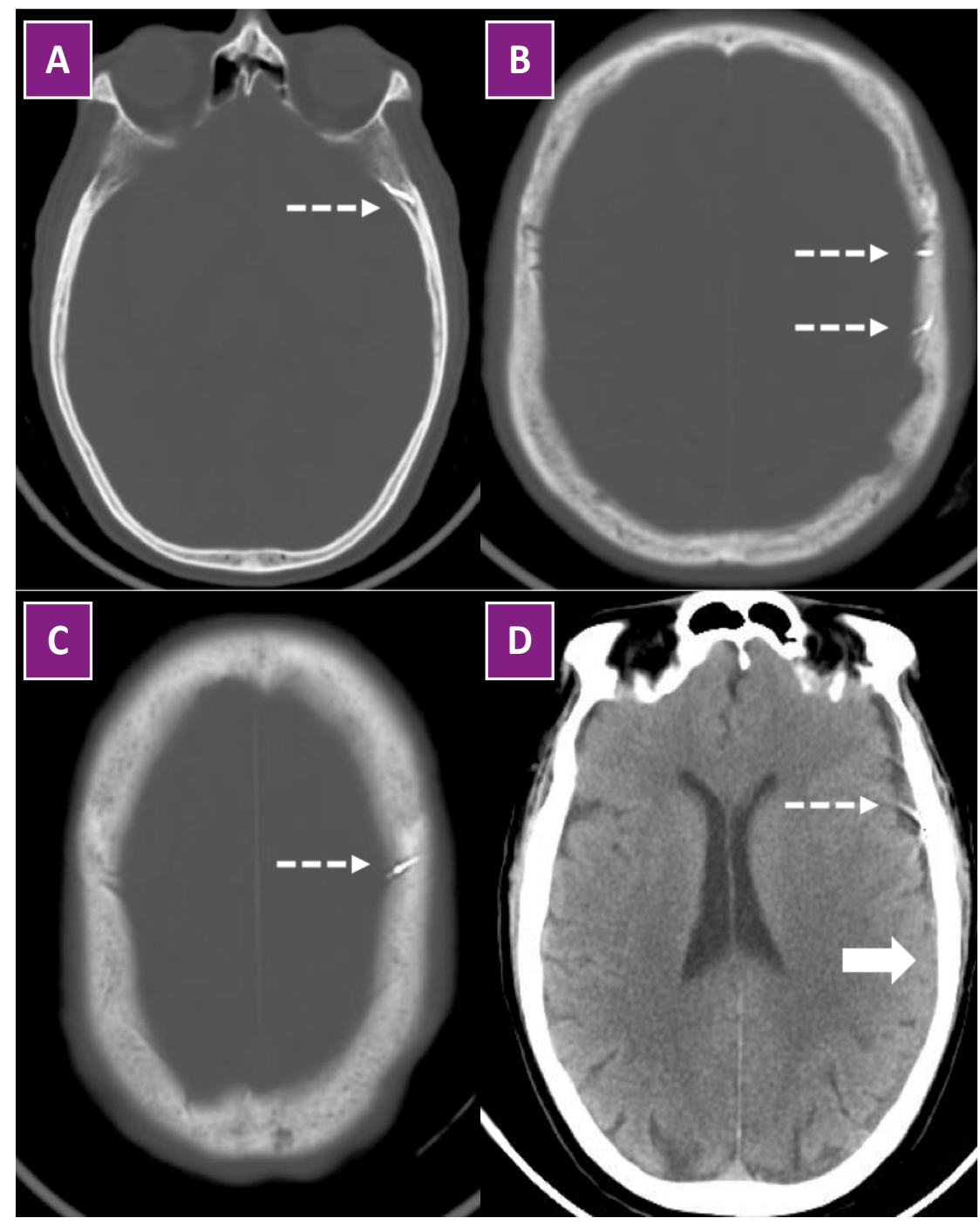

Figura 4. A, B y C: Tomografía espiral multicorte cerebral posoperatoria a los dos meses de la embolización, en el que se muestra la sustancia embolizante a nivel de la arteria meníngea media (flechas punteadas). D: Ausencia de hematoma subdural con reexpansión de parénquima cerebral (flecha gruesa) y presencia de material embolizante (flecha punteada).

Fuente de financiamiento: Autofinanciado.

Potencial conflicto de intereses: Los autores declaran no tener conflictos de intereses.

\section{ORCID}

John F. Vargas-Urbina, https://orcid.org/0000-0003-2437-8713 Giancarlo Saal-Zapata, https://orcid.org/0000-0002-4622-7118 Rodolfo Rodriguez-Varela, https://orcid.org/0000-0001-63613451

\section{REFERENCIAS BIBLIOGRÁFICAS}

1. Haldrup M, Ketharanathan B, Debrabant B, Søndergaard O, Mikkelsen R, Fugleholm K, et al. Embolization of the middle meningeal artery in patients with chronic subdural hematoma-a systematic review and meta-analysis. Acta Neurochir (Wien). 2020;162(4):777-84. doi: 10.1007/s00701-020-04266-0.

2. Martinez-Perez R, Rayo N, Tsimpas A. Embolización endovascular de la arteria meníngea media para el tratamiento de hematomas subdurales crónicos: efectividad, seguridad y controversia actual. Revisión sistemática. Neurología. 2020;S0213-4853(20)30133-X. doi: 10.1016/j.nrl.2020.04.023.

3. Almenawer SA, Farrokhyar F, Hong C, Alhazzani W, Manoranjan B, Yarascavitch B, et al. Chronic subdural hematoma management: a systematic review and meta-analysis of 34829 patients. Ann Surg. 2014;259(3):449-57. doi: 10.1097/SLA.0000000000000255.

4. Liu W, Bakker NA, Groen RJM. Chronic subdural hematoma: a systematic review and meta-analysis of surgical procedures. J Neurosurg. 2014;121(3):665-73. doi: 10.3171/2014.5.JNS132715.

5. Entezami P, Boulos A, Paul A, Nourollahzadeh E, Dalfino J. Contrast enhancement of chronic subdural hematomas after embolization of the middle meningeal artery. Interv Neuroradiol. 2019;25(5):596600. doi: $10.1177 / 1591019919843354$. 
6. Link TW, Boddu S, Marcus J, Rapoport BI, Lavi E, Knopman J. Middle meningeal artery embolization as treatment for chronic subdural hematoma: a case series. Oper Neurosurg (Hagerstown). 2018;14(5):556-562. doi: 10.1093/ons/opx154.

7. Link TW, Rapoport BI, Paine SM, Kamel H, Knopman J. Middle meningeal artery embolization for chronic subdural hematoma: Endovascular technique and radiographic findings. Interv Neuroradiol. 2018;24(4):455-462. doi: 10.1177/1591019918769336.

8. Wang Y, Zhou J, Fan C, Wang D, Jiao F, Liu B, et al. Influence of antithrombotic agents on the recurrence of chronic subdural hematomas and the quest about the recommencement of antithrombotic agents: A meta-analysis. J Clin Neurosci. 2017;38:79-83. doi: 10.1016/j.jocn.2016.12.001.

9. Qian Z, Yang D, Sun F, Sun Z. Risk factors for recurrence of chronic subdural hematoma after burr hole surgery: potential protective role of dexamethasone. Br J Neurosurg. 2017;31(1):84-88. doi: 10.1080/02688697.2016.1260686.

10. Wada M, Yamakami I, Higuchi Y, Tanaka M, Suda S, Ono J, et al. Influence of antiplatelet therapy on postoperative recurrence of chronic subdural hematoma: A multicenter retrospective study in 719 patients. Clin Neurol Neurosurg. 2014;120:49-54. doi: 10.1016/j.clineuro.2014.02.007.

11. Chon K-H, Lee J-M, Koh E-J, Choi H-Y. Independent predictors for recurrence of chronic subdural hematoma. Acta Neurochir (Wien).
Acta Neurochir (Wien). 2012;154(9):1541-8. doi: 10.1007/s00701012-1399-9.

12. Court J, Touchette CJ, Iorio-Morin C, Westwick HJ, Belzile F, Effendi K. Embolization of the Middle meningeal artery in chronic subdural hematoma - A systematic review. Clin Neurol Neurosurg. 2019;186:105464. doi: 10.1016/j.clineuro.2019.105464.

13. Mandai S, Sakurai M, Matsumoto Y. Middle meningeal artery embolization for refractory chronic subdural hematoma. J Neurosurg. 2000;93(4):686-8. doi: 10.3171/jns.2000.93.4.0686.

14. Tempaku A, Yamauchi S, Ikeda H, Tsubota N, Furukawa H, Maeda $D$, et al. Usefulness of interventional embolization of the middle meningeal artery for recurrent chronic subdural hematoma: five cases and a review of the literature. Interv Neuroradiol. 2015;21(3):366-71. doi: 10.1177/1591019915583224.

15. Hashimoto $T$, Ohashi T, Watanabe D, Koyama S, Namatame $H$ Izawa $\mathrm{H}$, et al. Usefulness of embolization of the middle meningeal artery for refractory chronic subdural hematomas. Surg Neurol Int. 2013;4:104. doi: 10.4103/2152-7806.116679.

16. Farkas N, Bo R, Arcot K, Tiwari A, Turkel-Parrella D, Selas G, et al. Radiographic efficacy of middle meningeal artery embolization in treatment of chronic subdural hematoma (P6.213). Neurology. 2018; 90(15 Supplement):P6.213. 